\title{
Genital warts: comparing clinical findings to dermatoscopic aspects, in vivo reflectance confocal features and histopathologic exam
}

\author{
John Verrinder Veasey ${ }^{1}$ \\ Valéria Maria de Souza Framil ${ }^{2}$ \\ Sidney Roberto Nadal ${ }^{3}$ \\ Alessandra Cristine Marta ${ }^{4}$ \\ Rute Facchini Lellis ${ }^{5}$
}

DOI: http://dx.doi.org/10.1590/abd1806-4841.20141917

\begin{abstract}
Genital warts can be diagnosed through physical examination and confirmed by histopathology. Noninvasive methods are useful for ruling out other diagnoses with no harm to the patient. In this study the clinical findings were compared to dermoscopy, reflectance confocal microscopy (RCM), and to histopathology findings, in order to determine possible patterns that can aid diagnosis of the lesion. It was possible to identify structural changes on reflectance confocal microscopy that are already known by dermoscopy, in addition to cellular changes previously seen only by histopathological examination. This study shows the use of reflectance confocal microscopy in cases of genital warts, providing important information that can be used in further studies.
\end{abstract}

Keywords: Condylomata acuminata; Dermoscopy; Microscopy, confocal

\section{INTRODUCTION}

Genital warts are sexually transmitted diseases (STD) caused by the human papillomavirus (HPV). It is considered to be the most frequently encountered STD in developing countries and can be classified as a global epidemic. ${ }^{1,2}$

Genital warts can normally be diagnosed by the naked eye. However, other conditions arising in the anogenital area such as seborrheic keratosis, lichen planus, condylomata lata and bowenoid papulosis should be ruled out. ${ }^{1,3}$

Dermatoscopy is a noninvasive, in vivo diagnostic technique which has proved to be especially valuable in examining pigmented skin lesions. Its use for evaluating non-pigmented skin lesions can reveal some characteristics not visible to the naked eye. Several authors have described the dermatoscopic findings of genital warts as having morphologic patterns (fingerlike, knoblike, mosaic-like) and vascular features. ${ }^{4,5}$

In vivo reflectance confocal microscopy (RCM) is a new non-invasive auxiliary imaging method based on an optic system consisting of a light source (diode laser of $830 \mathrm{~nm}$ wave length) and a set of objective lenses. The detector can process the light received showing images similar to histologic slides that repre- sent consecutive transversal slides of the skin. The images can reach a depth of up to 350um, allowing the visualization of the epidermis and superficial dermis. The in vivo evaluation of the skin is painless and shows the dynamic cell phenomena in real time. This can be crucial for the study of cancers. ${ }^{6}$ It allows analysis of the pigmented lesions and identification of the surgical margins, determining with cellular precision the whole area requiring removal. ${ }^{7,8}$ Some authors have also been using the RCM for evaluating infectious viral diseases such as molluscum contagiosum and varicella infections..$^{9,10}$

The main histological features of genital warts are well-known and easily identified on routine hematoxylin-eosin ( $\mathrm{H}$ \& E) staining: koilocytosis, papilomatosis and vascular distention. Langerhans' cells (LC) and melanocytes are both dendritic cells that are typically found within the spinous layer of the epidermis, and which often exhibit a stellate morphology. The LC vary in density within the human skin. On the head and neck, trunk, and extremities, LC can be found with a surface area up to 600 $1000 / \mathrm{mm}^{2}$. However, this can be as small as $200 / \mathrm{mm}^{2}$ on the palm, sole and anogenital skin. LC are antigen-

\section{Received on 08.06.2012.}

Approved by the Advisory Board and accepted for publication on 15.02.2013.

Work performed at the Dermatology Clinic, Irmandade da Santa Casa de Misericórdia de São Paulo, São Paulo (SP), Brazil.

Conflict of interest: None

Financial funding: None

MD; Dermatology Division, Department of Medicine, Santa Casa Medical School, São Paulo (SP), Brazil.

PhD; Dermatology Division, Department of Medicine, Santa Casa Medical School, São Paulo (SP), Brazil.

PhD; Coloproctology Division, Department of Surgery, Santa Casa Medical School, São Paulo (SP), Brazil.

MD; Department of Medicine, Dermatology Division, Department of Medicine, Santa Casa Medical School, São Paulo (SP), Brazil.

MD; Department of Pathology, Santa Casa Medical School, São Paulo (SP), Brazil. 
presenting cells for $\mathrm{T}$ lymphocytes and play an important role in the immune surveillance of the epidermis. ${ }^{11,12}$ LC can be seen with immunohistochemical stains using protein anti-bodies S100 and CD1a.

The objective of this study is to compare RCM findings of genital warts to the clinical presentation, dermatoscopic and histologic characteristics in order to determine possible patterns that can aid the diagnosis of the lesion, and to prove the usefulness of this method.

\section{METHOD}

Two male patients presenting genital warts for over one year were assessed through clinical evaluation, dermatoscopic examination and RCM analysis. The warts were then surgically extracted and sent to histologic exam.

Patient number 1 presented a $3 \mathrm{~cm}$ diameter genital wart located on the penis. Patient 2 presented a $4 \mathrm{~cm}$ diameter wart located on the pubis. The warts needed to be large enough (at least $2 \mathrm{~cm}$ diamenter) to make it possible to extract sufficient material for histopathologic tests. They also had to be located on a flat area so that the RCM could be handled steadily and not compromise the quality of the image.

The dermatoscopic images were taken with a digital camera (Cyber-shot DSC W610, Sony Corp) attached to a dermatoscopic lens (Dermlite Hybrid) with polarized lights, using alcohol gel as a contact interface to the skin.

RCM were performed with Vivascope $3000^{\circledR}$, manufactured by Lucid-Tech Rochester, NY, USA. All RCM imaging was performed according to a standardized imaging protocol, which includes acquisition of the images with a digital camera, a dermatoscopic camera, and the confocal microscope. Subsequently, the captured images were compared with the biopsy findings.

Both samples included in this study were biopsied after RCM imaging and sent to histopathological exam. Slides were prepared and stained with hematoxylin-eosin and then immunohistochemical reactions were performed with melan-A to evaluate the presence and distribution of melanocytes, and with CD1a to evaluate the same characteristics of Langerhans' cells. The results were compared in order to evaluate the applicability of RCM on the diagnosis of genital warts.

\section{RESULTS}

The clinical presentations of both genital warts are similar, presenting well-defined plaques with a rough surface, with no symptoms (Figure 1 ).

The dermatoscopic image of Patient 1 showed a fingerlike pattern, with relatively long, markedly separated fingerlike projections, similar in diameter but different in length. Patient 2 presented a knoblike pattern, with shorter, more rounded and closely aggregated knoblike projections similar in both diameter and length. The warts presented a rough aspect in both patterns. Single red dots were noted on the tip of many of the projections, corresponding to glomerular vessels in both cases (Figure 2).

Reflectance confocal microscopy basic image $(0.5 \times 0.5 \mathrm{~cm})$ of the spinous layer demonstrated a honeycomb pattern, with some larger cells that corresponded to koilocytosis. Deeper in the epidermis, at the dermoepidermal junction, the papillary dermis presented the glomerular vessels that were seen on dermatoscopy (Figures 3 and 4). Some areas showed a high number of dendritic cells, either Langerhans' cells or melanocytes, to be distinguished through histological examination.

The histopathologic study was essential for confirming the dermatoscopic and especially the RCM findings. The skin presented epidermic hyperplasia and papillomatosis. The superficial keratinocytes were enlarged, with a clear area around the nucleus, similar to koilocytosis. The papillary dermis presented dilated capillaries. The immunohistochemistry analysis distinguished the dendritic structures seen on some areas of the epidermis on RCM between melanocytes and LC. Melanocytes became positive on
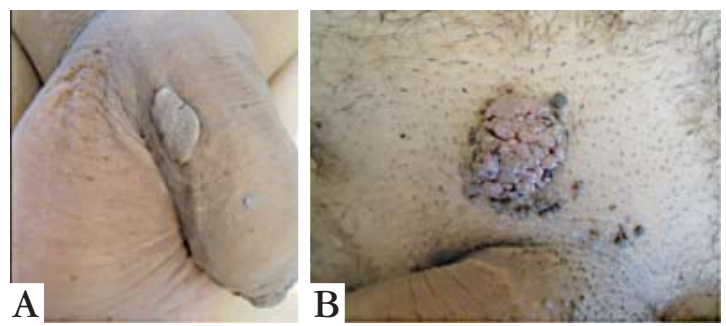

FIGURE 1: Clinical presentation of genital warts from Patient 1 (A) and 2 (B)
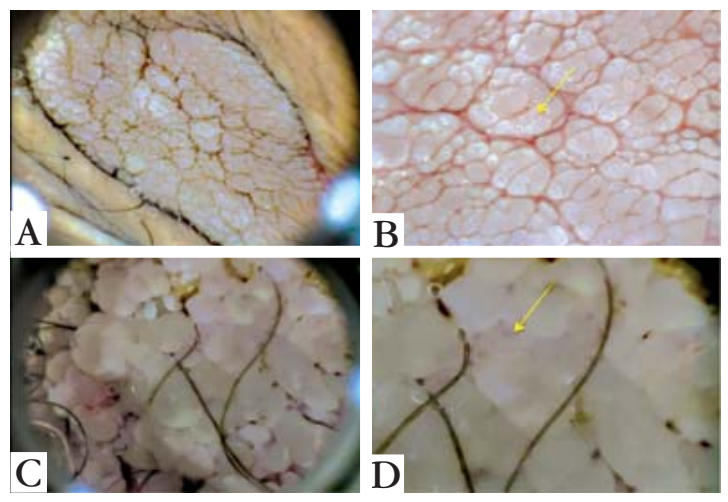

FIGURE 2: Dermoscopy of Patient 1 (A) showing fingerlike pattern and Patient 2 (C) with knoblike pattern. On images $\mathbf{B}$ and $\mathbf{D}$ it is possible to see glomerular vessels (arrowed) 

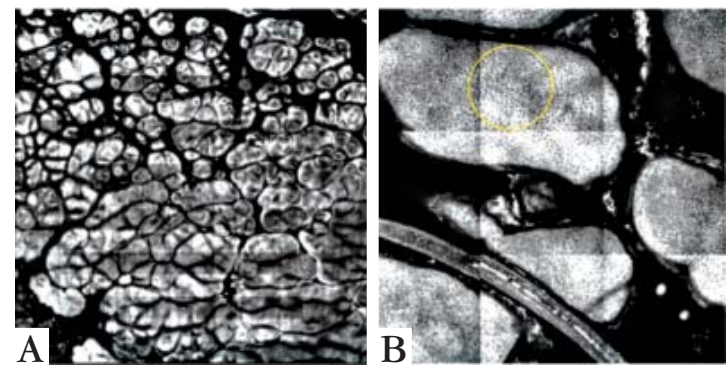

Figure 3: Papillomatosis of Patient 1 (A) in a $5 \times 5 \mathrm{~mm}$ view and of Patient 2 (B) in close-up, $1.5 \times 1.5 \mathrm{~mm}$, showing the honeycomb pattern of the spinous layer (circled)
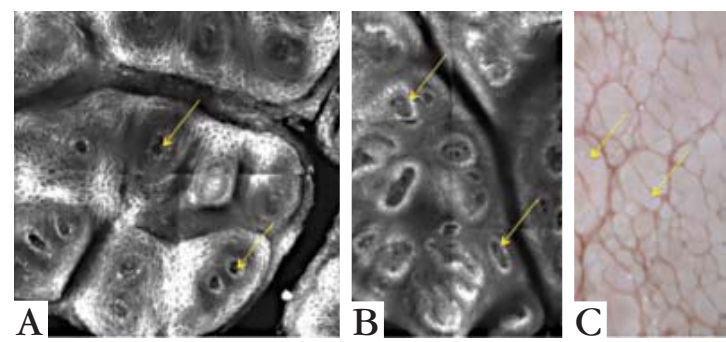

Figure 4: Comparing confocal microscopy findings with dermoscopy image. Confocal microscopy view of Patient 1 in a $1 \times 1 \mathrm{~mm}$ area (A) and of Patient B in a $1 \times 1.5 \mathrm{~mm}$ area (B). (C) is a dermoscopy picture. Arrows point to the vessels seen on confocal microscopy and on dermoscopy. The papillomatosis are seen on both exams, with a closer and better defined image on RCM

Melan-A reaction and distributed among the basal layer keratinocytes, whereas LC were positive on $\mathrm{CD} 1 \mathrm{a}$ reaction throughout the whole epidermis.

\section{DISCUSSION}

The dermatoscopic features of genital warts have already been fully described, with specific morphologic and vascular characteristics. The morphologic features may vary from a fingerlike to knoblike pattern, and the vascular pattern can be from glomerular to dotted. ${ }^{4,5}$ In this study, two warts were assessed. Some features seen by naked eye were also evident on dermatoscopy. The rough surfaces of the warts corresponded to papilomatosis on dermatoscopy and histopathology analysis. The dotted vessels of both patterns presented on dermatoscopy were also confirmed on histological examination.

RCM proved to be useful in the evaluation of the genital warts. The images of this method related to the dermatoscopy and histologic findings. The papilomatosis and vascular patterns were similar to the dermatoscopy and histological exam (Figure 3). It was also possible to identify the epidermic architecture variation in some areas, presenting koilocytosis and dendritic structures that were only identifiable by histopathologic exam. This evaluation was feasible due to the possibility of the RCM reaching up to 350 nanometers, allowing the observation of the epidermis and superficial dermis morphology (Figure 5).

Several researchers have demonstrated the usefulness of RCM in differentiating melanoma from benign pigmented lesions. ${ }^{6,7,8,13,14}$ These findings have also been related to dermatoscopy and histologic alterations. ${ }^{13,14}$ However, Hashemi et al have demonstrated that the diagnosis of melanoma using RCM can lead to wrong interpretations. Dendritic cells on the epidermis that were thought to be melanocytes in a pagetoid pattern on RCM were actually LC, marked by immunohistochemical stains. The histopathologic exam ended up proving the diagnosis of the lesions as benign nevi with melanocytes present only on the basal layer.

Langerhans' cells vary in density within the human skin. ${ }^{11}$ On the head, neck, trunk, and extremities, LC can be found on surfaces as large as 600 to $1000 / \mathrm{mm}^{2}$. However, this area can be as small as 200/ $\mathrm{mm}^{2}$ in the palm, sole and anogenital skin. ${ }^{12}$ In the present study, the RCM showed dendritic cells on the epidermis of both lesions. Histopathologic exams were performed with immunohistochemical stains to LC and melanocytes. Melanocytes were present only on the basal layer, while LC were evident throughout the epidermis (Figure 6). The density of dendritic cells on RCM was not measured for comparison with the density described in previous studies.
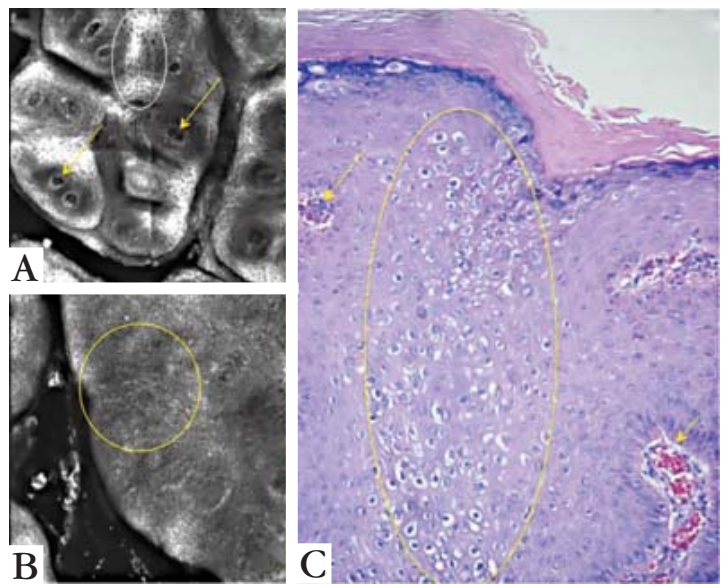

FIGURE 5: Comparing confocal microscopy findings with the histopathologic image. (A) is a $1 \times 1 \mathrm{~mm}$ view with vessels pointed out by the arrows. The same vessels are pointed on histologic image C. (B) is a $0.5 \times 0.5$ view showing the architectural disruption of the spinous layer without the usual honeycomb pattern. The disruption is seen on histologic image $\mathrm{C}$ as koilocytosis (yellow circles). On A it is also possible to see areas of the spinous layer with a honeycomb pattern (white circle) 

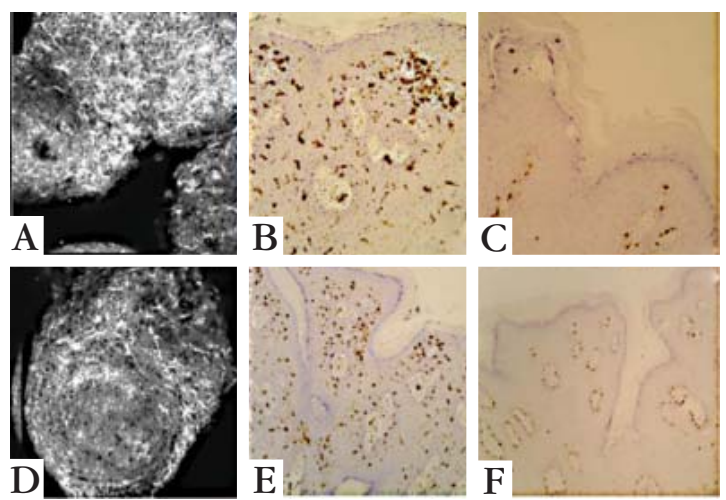

FiguRe 6: Images of Patient 1: confocal microscopy $0.5 \times 0.5 \mathrm{~mm}$ at spinous layer (A), histological exam with immunohistochemical reactions: CD1a (B) and melan-A (C). Images of Patient 2: confocal microscopy $0.5 \times 0.5 \mathrm{~mm}$ at spinous layer (D), histological exam with immunohistochemical reactions: CD1a (E) and melan-A (F). In both cases the white dendritic cells on RCM (A and D) were evident, and proved to be Langerhans cells with CD1a throughout the epidermis (B and E); melanocytes with melan-A were only present on the basal layer (C and $\mathbf{F})$
LC are antigen-presenting cells that participate in a highly specialized system, functioning as sentinel posts of the immune system, especially in peripheral non-lymphoid tissues that have a high antigenic exposure, such as the skin and mucosae. The presence of these cells in the wart may indicate the body's immune response to a foreign infection. ${ }^{11,12,14}$ However, $\mathrm{RCM}$ and histological exam was not done in normal non-infected skin of the genital area for comparison of the dendritic cells presentation, and the number of warts analyzed was not sufficiently large to come to any assumption.

In conclusion, the systematic comparison between clinical findings to dermatoscopic images and histologic exam, methods that are well-known and accepted in the evaluation of warts, helped us to interpret our RCM findings. Despite analysing only a few lesions, our study indicated the usefulness of in vivo reflectance confocal microscopy in skin disorders other than melanocytic lesions, and provided important information for use in further studies. $]$

\title{
REFERENCES
}

1. Forcier M, Musacchio N. An overview of human papillomavirus infection for the dermatologist: disease, diagnosis, management, and prevention. Dermatol Ther. 2010;23:458-76.

2. Anic GM, Giuliano AR. Genital HPV infection and related lesions in men. Prev Med. 2011;53:S36-41.

3. Leto MGP, Santos Jr GF, Porro AM, Tomimori J. Human papillomavirus infection: etiopathogenesis, molecular biology and clinical manifestations. An Bras Dermatol. 2011:86:306-17.

4. Dong H, Shu D, Campbell TM, Frühauf J, Soyer HP, Hofmann-Wellenhof R. Dermatoscopy of genital warts. J Am Acad Dermatol. 2011;64:859-64.

5. Watanabe T, Yoshida Y, Yamamoto 0. Differential diagnosis of pearly penile papules and penile condyloma acuminatum by dermoscopy. Eur $\mathrm{J}$ Dermatol. 2010;20:414-5.

6. Rito C, Pineiro-Maceira J. Reflectance confocal microscopy in the diagnosis of cutaneous melanoma. An Bras Dermatol. 2009; 84:636-42.

7. Di Giacomo THB, Santiago AVD, Braga JCT, Blumetti TCMP, Ferreira JASLB, Canosa JM, et al. Perspectivas no uso da microscopia confocal in vivo na prática do cirurgião dermatológico. Surg Cosmet Dermatol. 2011;3:338-44.

8. Masters B, So P. Confocal microscopy and multi-photon excitation microscopy of human skin in vivo. Opt Express. 2001;8:2-10.

9. Abraham LS, Costa MC, Agozzino M, Amorosi B, Cota C, Ardigo M. In vivo reflectance confocal microscopy for varicella prompt diagnosis and treatment in a severely immunosuppressed patient. Skin Res Technol. 2012;18:386-8.

10. Scope A, Benvenuto-Andrade C, Gill M, Ardigo M, Gonzalez S, Marghoob AA. Reflectance confocal microscopy of molluscum contagiosum. Arch Dermatol. 2008;144:134.

11. Enokihara MY, Sasso WS, Enokihara MMSS, Paschoal LHC. Contribuição ao estudo ultra-estrutural das celulas de Langerhans no condiloma acuminado. An Bras Dermatol. 1997;72:329-34.

12. Jimenez-Flores R, Mendez-Cruz R, Ojeda-Ortiz J, Muñoz-Molina R, BalderasCarrillo 0, de la Luz Diaz-Soberanes M, et al. High-risk human papilloma virus infection decreases the frequency of dendritic Langerhans' cells in the human female genital tract. Immunology. 2005;117:220-8.

13. Segura S, Puig S, Carrera C, Palou J, Malvehy J. Dendritic cells in pigmented basal cell carcinoma: a relevant finding by reflectance-mode confocal microscopy. Arch Dermatol. 2007;143:883-6

14. Hashemi P, Pulitzer MP, Scope A, Kovalyshyn I, Halpern AC, Marghoob AA. Langerhans cells and melanocytes share similar morphologic features under in vivo reflectance confocal microscopy: a challenge for melanoma diagnosis. J Am Acad Dermatol. 2012;66:452-62.

\author{
MAILING ADDRESS: \\ John Verrinder Veasey \\ Clínica de Dermatologia da Santa Casa de São Paulo \\ Rua Dr Cesário Mota Jr, 112 \\ Edifício Conde de Lara, $5^{\circ}$ andar - Santa Cecília \\ 01221-020 - São Paulo-SP \\ Brazil \\ E-mail: johnnyveasey@hotmail.com
}

How to cite this article: Veasey JV, Framil VMS, Nadal SR, Marta AC, Lellis RF. Genital warts: comparing clinical findings to dermatoscopic aspects, in vivo reflectance confocal features and histopathologic exam. An Bras Dermatol. 2013;89(1):137-40. 